\section{Comparison of Strongyloides- specific IgG enzyme immunoassays for laboratory detection of Strongyloides stercoralis infection}

Sir,

The soil-transmitted intestinal nematode, Strongyloides stercoralis (Strongyloides), the cause of strongyloidiasis, is a neglected tropical pathogen affecting approximately 30-100 million people worldwide. ${ }^{1}$ Reactivated strongyloidiasis in immunocompromised patients is of significant clinical concern because occult infection can potentially escalate to a life-threatening hyper-infection syndrome involving the multiorgan dissemination of larvae. ${ }^{1}$ With a growing immunosuppressed population, widespread travel and rising global immigration from endemic areas, Strongyloides infection has become an emerging health concern both in less-developed and developed countries, which is exacerbated by inaccurate laboratory diagnosis. ${ }^{1}$

Routine laboratory diagnosis of strongyloidiasis involves microscopy for parasite larvae in stool and serological assays for Strongyloides-specific immunoglobulin G (IgG). ${ }^{2}$ Among current serological assays, enzyme immunoassays (EIAs) that employ antigens of native crude parasite extracts (CrAgs) derived from either homologous or heterologous Strongyloides species, are the most commonly utilised assays in routine screening. ${ }^{2}$ However, CrAgs sourced from infected individuals or experimental animals are limited and cumbersome. ${ }^{1-4}$ More importantly, despite EIAs utilising CrAgs (CrAg-EIAs) exhibiting a higher sensitivity compared to conventional stool examinations, these assays suffer from suboptimal specificity, mainly due to cross-reactivity with antibodies produced against other co-circulating helminths. ${ }^{2-}$

${ }^{5}$ In low disease prevalence populations including mainstream Australian communities, the lack of highly specific assays also causes difficulty in achieving an accurate diagnosis of the Strongyloides infection, leading to false positives. In clinical practice, serology results often require careful interpretation in conjunction with clinical manifestations, epidemiological factors, and exposure histories. ${ }^{4}$

One recognised advance to address these drawbacks is the use of recombinant parasite antigens, such as a $31 \mathrm{kDa}$ protein (termed $\mathrm{NiE}$ ) derived from a $S$. stercoralis $\mathrm{L} 3$ parasite cDNA library, which are easily produced in large quantities and demonstrate improved specificity while maintaining comparable sensitivity. ${ }^{2-8}$ Given the numerous fastidious EIAs that are commercially available, this study compared three conventional CrAg-EIAs and a novel EIA that utilised the recombinant $\mathrm{NiE}$ antigen ( $\mathrm{rNiE}$ ) for serodiagnosis of Strongyloides infection in a tertiary reference laboratory setting.

Our retrospective study used 123 archived serum samples referred for Strongyloides testing between 2018 and 2020 previously shown as anti-Strongyloides IgG positive $(n=45)$, negative $(n=46)$ or equivocal $(n=32)$ using an initial $S$. rattibased in-house CrAg-EIA. Each sample was retested using four commercial assays, among which three utilised CrAgs: the Bordier $S$. ratti ELISA (Bordier Affinity Products, Switzerland), the Euroimmun Anti-Strongyloides ELISA (Euroimmun, Germany) based on S. papillosus, and the DRG
Strongyloides IgG ELISA (DRG Instruments, Germany) based on homologous $S$. stercoralis. The fourth assay, the InBios StrongyDetect IgG ELISA (InBios International, USA), employed rNiE and was released for research only. All testing protocols followed the manufacturer's kit instructions. Fixed cut-off values were available from the manufacturers for all three CrAg-EIA kits. The manufacturer of the InBios kit recommended that end users determine cut-off values de novo using sufficient numbers of well-characterised local serum samples. The initial cut-off value for the InBios kit was proposed at optimal density $(\mathrm{OD})=0.178$, as published previously.

Statistical analysis was performed to estimate agreement measures using SPSS Statistics software (SPSS, USA): positive percent agreement (PPA), negative percent agreement (NPA), overall percent agreement (OPA) and Cohen's kappa statistic $(k)$. Level of significance at $95 \%$ confidence interval (CI) was reported for each measure. The Cohen's kappa coefficient is a robust statistical metric to assess the level of agreement (beyond chance) between two tests. Based on the $k$ value, the agreement was defined as poor $(<0.00)$, slight $(0.00-0.20)$, fair $(0.21-0.40)$, moderate $(0.41-0.60)$, substantial $(0.61-0.80)$, and almost perfect $(0.81-1.00)$. These measures were computed for each of three index CrAg-EIA kits and the novel InBios kit that employed rNiE, compared to a composite reference standard defined as consensus results obtained from at least two of the three CrAg-EIA kits to evaluate their diagnostic performance. In the absence of gold standard, using consensus results from multiple tests allows an improved classification of true disease status of the study samples. Pairwise comparisons between test kits by Cohen's kappa statistic were also performed.

An equivocal (indeterminate) range was provided only for one of the CrAg-based EIA kits (Euroimmun). Samples testing equivocal for this assay $(n=17)$ were excluded from statistical analysis. Based on the composite reference standard, 24/106 samples were classified as anti-Strongyloides IgG positive and 82/106 classified as negative. Table 1 shows the extent to which the three CrAg-EIA kits and the novel InBios $\mathrm{rNiE}$ kit agreed with the composite reference standard.

Among the selected commercial CrAg-EIA kits, the Bordier kit has been reported frequently to have a high diagnostic sensitivity from $83 \%$ to $91 \%$ and specificity ranging from $89 \%$ to $98 \% .8,10,11$ The data from our study were comparable with previous studies, the kit exhibiting adequate performance with 95.8\% PPA (95\% CI 79.8-99.3\%) and $92.7 \%$ NPA (95\% CI 84.9-96.9\%), with $93.4 \%$ OPA (95\% CI 87-96.8\%) and an almost perfect kappa value at $k=0.82(p<0.0005,95 \%$ CI $0.64-0.95)$. Nonetheless, despite its high-performance parameters, published studies also observed frequent false positive reactions when using the Bordier kit among sera from patients suffering from other parasitic infections (11.3\%), particularly with filarial species $(37.8 \%)$.

The Euroimmun assay utilises purified native antigens from $S$. papillosus, aiming for improved specificity compared to CrAgs using $S$. ratti-derived antigens. A manufacturerindependent study to evaluate the performance of the Euroimmun assay and the suitability of using $S$. papillosus-derived antigen has not been published. Compared with the Bordier kit, the Euroimmun kit exhibited an improved PPA of $100 \%$ (95\% CI 86.2-100\%), NPA of $96.3 \%$ (95\% CI 89.8-98.7\%), OPA of $97.2 \%$ (95\% CI 92-99\%) and Cohen's kappa $k=0.92$ 
Table 1 Comparisons of four Strongyloides-specific IgG EIA kits to the composite reference standard for the serodiagnosis of Strongyloides infection

\begin{tabular}{|c|c|c|c|c|c|c|c|}
\hline \multirow[t]{2}{*}{ EIAs } & \multirow[t]{2}{*}{ Test result } & \multirow[t]{2}{*}{$\begin{array}{c}\text { Positive } \\
n\end{array}$} & \multirow[t]{2}{*}{$\begin{array}{c}\text { Negative } \\
n\end{array}$} & \multicolumn{4}{|c|}{$\begin{array}{l}\text { Composite reference standard } \\
\text { (consensus results obtained from at least two of the three CrAg-EIA kits) }\end{array}$} \\
\hline & & & & $\begin{array}{l}\text { Positive \% agreement } \\
\quad(95 \% \mathrm{CI})\end{array}$ & $\begin{array}{l}\text { Negative \% agreement } \\
\qquad(95 \% \mathrm{CI})\end{array}$ & $\begin{array}{l}\text { Overall \% agreement } \\
\qquad(95 \% \mathrm{CI})\end{array}$ & $\begin{array}{l}\text { Cohen's kappa index } k \\
(p \text { value, } 95 \% \mathrm{CI})\end{array}$ \\
\hline Bordier & Positive & 23 & 6 & $95.8 \%$ & $92.7 \%$ & $93.4 \%$ & 0.82 \\
\hline S. ratti-based CrAg & Negative & 1 & 76 & $(79.8-99.3 \%)$ & $(84.9-96.9 \%)$ & $(87-96.8 \%)$ & $(p<0.0005,0.64-0.95)$ \\
\hline S. papillosus-based CrAg & Negative & 0 & 79 & $(86.2-100 \%)$ & $(89.8-98.7 \%)$ & $(92-99 \%)$ & $(p<0.0005,0.83-1.00)$ \\
\hline DRG & Positive & 12 & 0 & $50 \%$ & $100 \%$ & $88.7 \%$ & 0.61 \\
\hline S. stercoralis-based $\mathrm{CrAg}$ & Negative & 12 & 82 & $(31.4-68.6 \%)$ & $(95.5-100 \%)$ & $(81.2-93.4 \%)$ & $(p<0.0005,0.38-0.84)$ \\
\hline InBios & Positive & 13 & 4 & $54.2 \%$ & $95.1 \%$ & $85.8 \%$ & 0.55 \\
\hline $\mathrm{rNiE}$ & Negative & 11 & 78 & $(35.1-72.1 \%)$ & $(88.1-98.1 \%)$ & $(78-91.2 \%)$ & $(p<0.0005,0.26-0.73)$ \\
\hline
\end{tabular}

CI, confidence interval; CrAg-EIAs, enzyme immunoassays (EIAs) that employ native crude antigen extracts of the parasite (CrAgs); rNiE, recombinant NiE antigen.

Cohen's kappa coefficient $(k)$ indicates the level of agreement as follows: poor $k<0.00$, slight $0.00-0.20$, fair $0.21-0.40$, moderate $0.41-0.60$, substantial $0.61-$ 0.80 , and almost perfect $0.81-1.00$

$(p<0.0005,0.83-1.00)$ with reference to the composite reference standard (Table 1). As illustrated in Table 2, substantial agreement was observed between the Euroimmun and Bordier kits in pairwise comparisons $(k=0.76, p<0.0005,95 \%$ CI 0.61-0.90). Overall, the Euroimmun kit exhibited comparable performance to that of the Bordier kit.

The DRG assay is a CrAg-EIA using homologous $S$. stercoralis species, that is reported to perform with specificity similar to or better than CrAgs prepared from heterologous Strongyloides species. ${ }^{8,10,11}$ However, in our study, the DRG kit exhibited a lower PPA of 50\% (95\% CI 31.4$68.6 \%)$ and a lower agreement with the composite reference standard $(k=0.61 ; p<0.0005,95 \%$ CI $0.38-0.84)$, indicating a lower sensitivity. This is the first study that evaluated the performance of the DRG kit for serodiagnosis of Strongyloides infection. Due to this extremely low PPA, caution should be used while interpreting results, despite it exhibiting $100 \%$ NPA.

When compared to a composite reference standard constructed using the three commercial CrAg-EIAs, the InBios kit, which uses the rNiE antigen, yielded a high NPA of $95.1 \%$ (95\% CI 88.1-98.1\%) which was comparable to that of the Bordier and Euroimmun kits. However, a considerable proportion of samples $(n=11)$ returned contradictory negative results on this kit. This resulted in a lower PPA of $54.2 \%(95 \%$ CI $35.1-72.1 \%$ ) and moderate agreement to the composite reference standard at $k=0.55(p<0.0005,95 \%$ CI $0.26-0.73)$. Moderate agreement was also observed when comparing this kit with the Bordier assay $(k=0.46, p<0.0005,95 \%$ CI $0.26-$ $0.64)$ and with the Euroimmun kit $(k=0.49, p<0.0005,95 \%$ CI $0.27-0.68$ ), as shown in Table 2 . Using the same cut-off value and faecal examination to define a reference standard, previous authors reported contradictory performance for the InBios assay, with a higher sensitivity of $82.9 \%$ and a lower specificity of $57.9 \%$. Moreover, more homogenous performance parameters of the InBios kit were demonstrated in a study evaluating the kit against the consensus results obtained from at least two of three index assays (PPA 83.6\%, NPA 91.3\%, $k=0.74$ ), LIPS (luciferase immunoprecipitation systems) assay (PPA 74.1\%, NPA 78.7\%, $k=0.53$ ), and stool culture (sensitivity $80 \%$, specificity $90 \%) .{ }^{12}$ The published studies regarding the diagnostic performance of this kit are not only limited in numbers but also the available data vary substantially due to differences in study population, sample size and the reference standard employed. Currently, the lack of manufacturer provided cut-off values limits verification and application of the InBios assay, especially in areas where abundant well-characterised serum samples are difficult to obtain. Therefore, the InBios kit may be useful only for research purposes. In addition, a potential limitation of our study is the use of the composite reference standard as it carries a risk of misclassifying samples, which can result in over-

Table 2 Pairwise comparisons of results of four Strongyloides-specific IgG EIA kits (Cohen's kappa index $k$ )

\begin{tabular}{|c|c|c|c|c|}
\hline & $\begin{array}{c}\text { Bordier } \\
(p \text { value, } 95 \% \mathrm{CI})\end{array}$ & $\begin{array}{c}\text { Euroimmun } \\
(p \text { value, } 95 \% \mathrm{CI})\end{array}$ & $\begin{array}{c}\text { DRG } \\
(p \text { value, } 95 \% \mathrm{CI})\end{array}$ & $\begin{array}{c}\text { InBios } \\
(p \text { value, } 95 \% \mathrm{CI})\end{array}$ \\
\hline
\end{tabular}

$\mathrm{CI}$, confidence interval; $\mathrm{CrAg}-\mathrm{Ags}$, native crude antigen extracts of the parasite; rNiE, recombinant NiE antigen. 
estimates of false positivity or false-negative results in the test assays.

In conclusion, among the three CrAg-EIAs within the study, the Bordier and Euroimmun kits appear to exhibit similar performance characteristics as competitive serological tools compared to the DRG assay. The introduction of the InBios EIA as a more specific alternative to replace the existing CrAg-based EIAs remains difficult in this first-time evaluation, due to the lack of well-defined cut-off values in different populations. Importantly, the data presented here are indicative of the need for further prospective studies to be conducted on a large scale, well-characterised population, with an emphasis on the $\mathrm{rNiE}$ antigen, provided that studies broadly support its enhanced specificity. This would meet the objective of improving the accuracy of Strongyloides serodiagnosis in the Australian population.

Acknowledgement: We thank the laboratory scientists of the Serology and Virology Division (SAViD) at the NSW Health Pathology SEALS Randwick for technical assistance, and the NSW Organ and Tissue Donation Service (OTDS) for collaboration on this study.

Conflicts of interest and sources of funding: The study is supported by the NSW Organ and Tissue Donation Service (OTDS). The authors state that there are no conflicts of interest to disclose.

\section{Yonghui $\mathbf{L u}^{1}$, Vidiya Ramachandran ${ }^{2}$, Siddhartha Mahanty ${ }^{3}$, William D. Rawlinson ${ }^{1,2, *}$}

${ }^{1}$ Serology and Virology Division (SAViD), NSW Health Pathology Randwick, Level 4, Prince of Wales Hospital, Randwick, NSW, Australia; ${ }^{2}$ School of Medical Sciences and School of Women's and Children's Health, Faculty of Medicine, School of Biotechnology and Biomolecular Sciences, Faculty of Science, University of New South Wales, Randwick, NSW, Australia; ${ }^{3}$ The Peter Doherty Institute for Infection and Immunity, Department of Infectious Diseases, Melbourne Medical School, University of Melbourne, Melbourne, Vic, Australia; *joint senior author

Contact Prof William D. Rawlinson.

E-mail: w.rawlinson@unsw.edu.au

1. Vasquez-Rios G, Pineda-Reyes R, Pineda-Reyes J, et al. Strongyloides stercoralis hyperinfection syndrome: a deeper understanding of a neglected disease. J Parasit Dis 2019; 43: 167-75.

2. Buonfrate D, Formenti F, Perandin F, et al. Novel approaches to the diagnosis of Strongyloides stercoralis infection. Clin Mcirobiol Infect 2015; 21: 543-52.

3. Krolewiecki AJ, Ramanathan R, Fink V, et al. Improved diagnosis of Strongyloides stercoralis using recombinant antigen-based serologies in a community-wide study in northern Argentina. Clin Vaccine Immunol 2010; 17: 1624-30.

4. Requena-Mendez A, Chiodini P, Bisoffi Z, et al. The laboratory diagnosis and follow up of strongyloidiasis: a systematic review. PLoS Negl Trop Dis 2013; 7: e2002.

5. Fradejas I, Herrero-Martinez JM, Lizasoain M, et al. Comparative study of two commercial tests for Strongyloides stercoralis serologic diagnosis. Trans R Soc Trop Med Hyg 2018; 112: 561-7.

6. Ravi V, Ramachandran S, Thompson RW, et al. Characterization of a recombinant immunodiagnostic antigen (NIE) from Strongyloides stercoralis L3-stage larvae. Mol Biochem Parasitol 2002; 125: 73-81.

7. Ramanathan R, Burbelo PD, Groot S, et al. A luciferase immunoprecipitation systems assay enhances the sensitivity and specificity of diagnosis of Strongyloides stercoralis infection. J Infect Dis 2008; 198 $444-51$.

8. Bisoffi Z, Buonfrate D, Sequi M, et al. Diagnostic accuracy of five serologic tests for Strongyloides stercoralis infection. PLoS Negl Trop Dis 2014; 8: e2640.

9. Ruantip S, Eamudomkarn C, Techasen A, et al. Accuracy of urine and serum assays for the diagnosis of strongyloidiasis by three enzymelinked immunosorbent assay protocols. Am J Trop Med Hyg 2019; 100: $127-9$.

10. Van Doorn HR, Koelewijn R, Hofwegen H, et al. Use of enzyme-linked immunosorbent assay and dipstick assay for detection of Strongyloides stercoralis infection in humans. J Clin Microbiol 2007; 45: 438-42.

11. Bon B, Houze S, Talabani $\mathrm{H}$, et al. Evaluation of a rapid enzyme-linked immunosorbent assay for diagnosis of strongyloidiasis. J Clin Microbiol 2010; 48: 1716-9.

12. Anderson NW, Klein DM, Dornink SM, et al. Comparison of three immunoassays for detection of antibodies to Strongyloides stercoralis. Clin Vaccine Immunol 2014; 21: 732-6.

DOI: https://doi.org/10.1016/j.pathol.2021.03.002

\section{HMGCR autoantibody testing: two tiers required}

Sir,

Autoimmune myopathies are a group of immune-mediated muscle diseases classifiable by clinicopathological patterns. The different patterns have been increasingly shown to associate with various antigen-specific autoantibodies. Recently, the 3-hydroxy-3-methylglutaryl-coenzyme A reductase (HMGCR) autoantigen was added to others in a qualitative commercial line blot (Euroline Autoimmune Inflammatory Myopathies $16 \mathrm{Ag}$ et $\mathrm{cN}-1 \mathrm{~A}$ et HMGCR $\mathrm{IgG}$; Euroimmun, Germany) allowing more convenient screening for 18 different myositis-specific autoantibodies. ${ }^{2}$ Before introduction, all laboratories are required to verify assay performance in their local population using methods and analyses relevant to the assay's intended use. . $^{3,4}$

In practice the detection of myositis-specific antibodies (MSA) is used in the diagnostic work-up of suspected autoimmune myositis. The original labour-intensive immunoprecipitation assays ${ }^{5}$ have been largely replaced by technically simpler automatable assays amenable to high throughput. Line blots of multiple individual antigens adsorbed to a solid phase matrix allow rapid and simultaneous screening.

Autoantigens adsorbed to solid matrices may be presented in a distorted conformation when compared with tissueexpressed autoantigens and those that remain in fluid phase. This may impact assay performance. The manufacturer of the Euroline blot has recommended that, where available and depending on the antigen, users seek further evidence of positivity using indirect immunofluorescence (IIF) staining on Hep-2 cells and/or primate tissues. ${ }^{2}$ While lacking analytical specificity, the finding of an IIF staining pattern known to be consistent with the cellular distribution of the relevant myositis-specific autoantigen adds weight to the detection of disease-specific autoantibodies. However, data regarding their frequency of detection using these methods is generally lacking, which may limit diagnostic utility. ${ }^{1}$ Alternative methods for HMGCR autoantibody detection include IIF staining on liver substrate (HMGCR associated liver IFL pattern, or HALIP) ${ }^{6}$ and immunoprecipitation. ${ }^{7,8}$ 